Article

\title{
Bioanalytical Method Development Using Liquid Chromatography with Amperometric Detection for the Pharmacokinetic Evaluation of Forsythiaside in Rats
}

\author{
Yu-Tse Wu ${ }^{1, *}$, Meng-Ting Cai ${ }^{1}$, Chih-Wei Chang ${ }^{1}$, Ching-Chi Yen ${ }^{1}$ and Mei-Chich Hsu ${ }^{2}$ \\ 1 School of Pharmacy, Kaohsiung Medical University, Kaohsiung 807, Taiwan; \\ cappricio2012@gmail.com (M.-T.C.); wxes9050304@gmail.com (C.-W.C.); date0315@hotmail.com (C.-C.Y.) \\ 2 Department of Sports Medicine, Kaohsiung Medical University, Kaohsiung 807, Taiwan; \\ meichich@kmu.edu.tw \\ * Correspondence: ytwu@kmu.edu.tw; Tel.: +886-7-312-1101 (ext. 2254)
}

Academic Editor: Derek J. McPhee

Received: 31 August 2016; Accepted: 13 October 2016; Published: 16 October 2016

\begin{abstract}
An analytical method entailing high-performance liquid chromatography coupled with electrochemical detection was developed for determining forsythiaside (FTS) in rat plasma. Rat plasma samples were prepared through efficient trichloroacetic acid deproteination. FTS and the internal standard were chromatographically separated on a reversed-phase core-shell silica C18 column $(100 \mathrm{~mm} \times 2.1 \mathrm{~mm}$, i.d. $2.6 \mu \mathrm{m})$, with a mobile phase consisting of an acetonitrile- $0.05-\mathrm{M}$ phosphate solution (11.8:88.2, v/v), at a flow rate of $400 \mu \mathrm{L} / \mathrm{min}$. The calibration curve, with $r^{2}>0.999$, was linear in the $20-1000 \mathrm{ng} / \mathrm{mL}$ range. The intra- and interday precision were less than $9.0 \%$, and the accuracy ranged from $94.5 \%$ to $106.5 \%$ for FTS. The results indicated that the newly developed HPLC-EC method is more sensitive than previous reported methods using UV detection, and this new analytical method is applied successfully for the pharmacokinetic study of FTS. The hydrogel delivery system can efficiently improve bioavailability and mean residual time for FTS, as evidenced by the 2.5- and 6.3-fold increase of the area under the curve and the extension of the half-life, respectively.
\end{abstract}

Keywords: forsythiaside; amperometric detection; pharmacokinetics; thermoresponsive hydrogel

\section{Introduction}

Forsythiaside (FTS, Figure 1), a phenylethanoid glycoside, is a major ingredient isolated from Fructus Forsythiae [1]. This herb, called Lian-Qiao in traditional Chinese medicine (TCM), has been widely used to treat different infectious diseases, such as upper respiratory tract complaints caused by bacteria and viruses [2]. Shuang-Huang-Lian powder injection, a preparation containing extracts from Fructus Forsythiae is indicated to treat infectious diseases in TCM [3]. FTS has been used for preserving neuronal structure and function through the reduction of oxidative stress, a major strategy to treat Alzheimer disease and dementia [4]. In addition, FTS has been proposed as a possible treatment for chronic inflammatory conditions caused by the influenza A virus by suppressing the release of chemokine (C-C motif) ligand 5 (also known as RANTES) from human bronchial epithelial cells [5]. FTS also possesses antibacterial activity against Escherichia coli, Pseudomonas aeruginosa, and Staphylococcus aureus. The minimal inhibitory concentration of FTS for S. aureus is even lower than that for tetracycline [6]. 
<smiles>CC1OC(OCC2OC(OCCc3ccc(O)c(O)c3)C(O)[C@H](O)C2OC(=O)/C=C/c2ccc(O)c(O)c2)[C@H](O)[C@H](O)[C@H]1O</smiles>

(A)

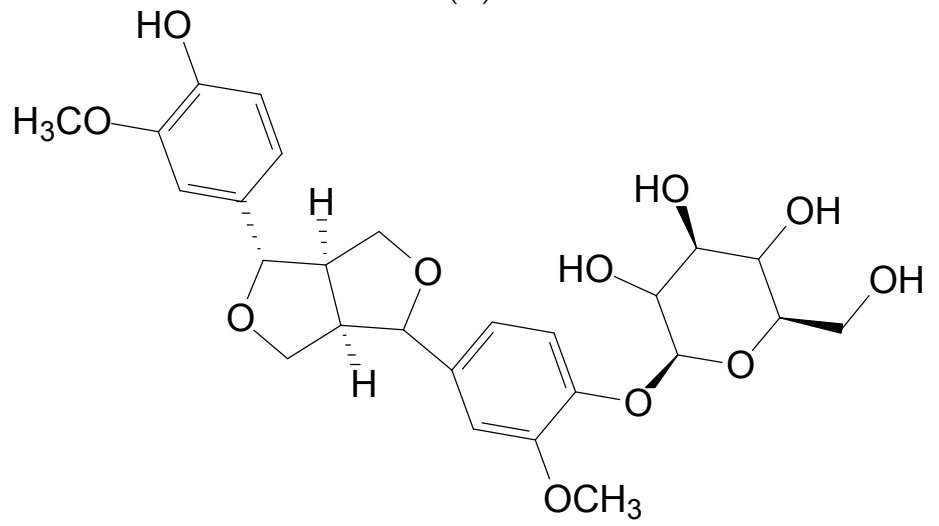

(B)

Figure 1. Chemical structure of (A) forsythiaside and (B) pinoresinol as the internal standard.

The pharmacokinetic evaluation of herbs is imperative because studies involving laboratory animals will provide useful information for the development of TCM, which in turn would facilitate the further evaluation of preclinical toxicological results of TCM for extrapolation to humans. In addition, during the early screening stages, evaluations of pharmacokinetics and bioavailability of potential lead compounds would become increasingly crucial if the main functional assays are molecule-based approaches, such as isolated protein assays or phenotypic assays using cells or model organisms [7]. Analytical methods with better sensitivity can collect more comprehensive time-concentration results and thus enhance the validity of the pharmacokinetic data [8]. Several pharmacokinetic studies of FTS have utilized high-performance liquid chromatography with ultraviolet detection (HPLC-UV) for analyzing rat and beagle dog plasma samples [1,9-13]. Similarly, HPLC with mass spectrometry (HPLC-MS) has been proposed for the pharmacokinetic evaluations of FTS [2,14-17]. HPLC-MS provides excellent selectivity and sensitivity but susceptible to matrix effects, that is, instable ionization efficiency caused by the competition between analytes and co-eluted components from the sample matrices, including phospholipids in plasma and additives to the mobile phases [18]. HPLC coupled with electrochemical detection (HPLC-EC) is an attractive alternative for determining FTS because it provides higher selectivity and sensitivity than does HPLC-UV and because it is not susceptible to matrix effects [19]. Pharmacokinetic studies have demonstrated that the half-life $\left(t_{1 / 2}\right)$ of FTS after intravenous administration ranges from 23.6 to $72.6 \mathrm{~min}$ in rats [13] and 64.3 to $89.4 \mathrm{~min}$ in beagle dogs [9]. The elimination $t_{1 / 2}$ of protein unbound FTS was measured to be $12.7 \pm 3.4$ and $18.2 \pm 5.5 \mathrm{~min}$ for rat blood and bile, respectively [1]. The therapeutic effectiveness and outcomes usually depend on whether the appropriate drug concentrations are achieved. In addition, therapeutic agents with short a half-life will need frequent administration to maintain the therapeutic effects. 
FTS possesses antimicrobials effects against infectious diseases, but the major hindrances to its clinical applicability are its low oral bioavailability (approximately $0.5 \%$ ) and rapid elimination, which may result in insufficient drug concentration in systemic blood circulation after drug administration. To prolong the $t_{1 / 2}$ of drugs, the in situ forming depot formulations are an ideal choice instead of frequent administration or constant infusion [20]. The in situ formation depot formulations has been proposed and investigated for controlled drug delivery in systemic treatments and localized therapies, and this formulation remains aqueous with a low viscosity but turns into a semisolid or solid depot after administration. The thermally-induced gelling hydrogel system is a type of in situ forming depot formulations, and is considered an intelligent drug-delivery system because it transitions from solution to gel conditions in response to an increase in the surrounding temperature [21]. Hydrogel drug-delivery systems are multicomponent systems consisting of a three-dimensional network of polymer chains and water molecules that fill the space between the macromolecules [22]. With such structural characteristics, hydrogels can sense the surrounding stimuli and respond by altering their chemical or physical status, leading to the controlled release of embedded active ingredients. In this study, we developed and validated a HPLC-EC method with an efficient protein precipitation pretreatment to determine FTS concentrations in rat plasma. The method was further applied to the in vivo pharmacokinetic characterization of FTS. Further, we designed an extended delivery formulation utilizing thermally-induced gelling hydrogel to solve the relatively short in vivo $t_{1 / 2}$ for FTS. The FTS-loaded hydrogel formulations were optimized according to the sol-gel transition temperature $\left(\mathrm{T}_{\text {sol-gel }}\right)$, temperature-viscosity profiles, and gel dissolution-drug release correlations.

\section{Results}

\subsection{Bioanalytical Method Validation for FTS}

\subsubsection{Selectivity}

Analytical method selectivity was examined by analyzing blank plasma for six different rats. No obvious interference peaks appeared around the retention times of FTS (13.6 min) and IS (10.7 min), as shown in the typical chromatograms (Figure 2). FTS is a caffeoyl glycoside consists of caffeic acid, 3,4-dihydroxy- $\beta$-phenethyl alcohol, D-glucose and L-rhamnose. The two genins and the two sugar moieties are polar and less retained on the reversed-phase stationary phase, and should appear before FTS in the chromatogram. The validation results indicated that the current method was reproducible and reliable. Therefore, the interference caused by metabolites of FTS is minimal and can be ignored.
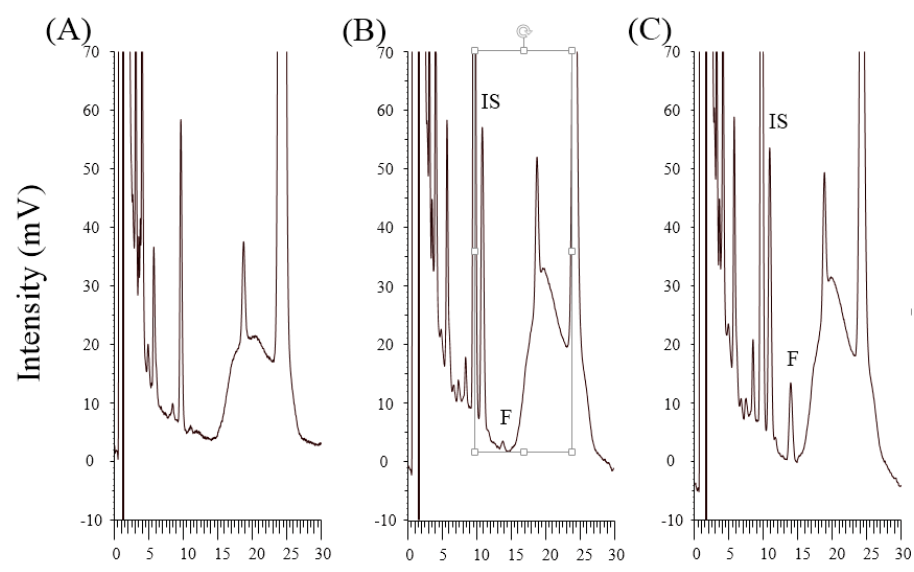

Retention time (min)

Figure 2. HPLC-EC chromatograms of (A) blank rat plasma; (B) blank plasma spiked forsythiaside $(20 \mathrm{ng} / \mathrm{mL})$ and $(\mathbf{C})$ plasma sample collected $15 \mathrm{~min}$ after subcutaneous injection of forsythiaside hydrogel (3 mg/kg). 


\subsubsection{Linearity and Sensitivity}

The linear regression equation of FTS was $\mathrm{y}=0.0009 \mathrm{x}-0.0031$, and all $r^{2}$ values were greater than 0.999 , which confirmed good linearity over the concentration range (20-1000 ng/mL). The limit of detection (LOQ) and (LOD) for FTS were 20 and $10 \mathrm{ng} / \mathrm{mL}$, respectively.

\subsubsection{Recovery}

Recovery $(n=3)$ for FTS was found to be $86.2 \% \pm 5.1 \%, 83.4 \% \pm 2.0 \%$ and $79.5 \% \pm 3.6 \%$ for low quality control (LQC: $40 \mathrm{ng} / \mathrm{mL}$ ), middle quality control (MQC: $400 \mathrm{ng} / \mathrm{mL}$ ), and high quality control (HQC: $750 \mathrm{ng} / \mathrm{mL}$ ) samples, respectively. The recovery for ISTD was $82.1 \% \pm 5.7 \%$.

\subsubsection{Accuracy and Precision}

The intraday accuracy of FTS ranged between $94.5 \%$ and $106.5 \%$, and the inter-day accuracy ranged between $96.6 \%$ and $99.8 \%$ (Table 1). The intra- and inter-day precisions, expressed as RSD, for FTS in rat plasma samples were found to be less than $7.5 \%$ and $9.0 \%$, respectively (Table 1), which confirmed the high precision of the developed method.

Table 1. Accuracy and precision of QC samples for FTS analysis.

\begin{tabular}{cccc}
\hline Nominal Concentration $(\mathbf{n g} / \mathbf{m L})$ & Observed Concentration $\mathbf{( n g / m L )}$ & Precision $(\mathbf{\%})$ & Accuracy $(\%)$ \\
\hline Intra-day & & & \\
20 & $18.2 \pm 1.2$ & 6.6 & $91.2 \pm 6.0$ \\
40 & $42.6 \pm 2.8$ & 6.6 & $106.5 \pm 7.0$ \\
400 & $378.0 \pm 28.3$ & 7.5 & $94.5 \pm 7.1$ \\
750 & $761.2 \pm 18.4$ & 2.4 & $101.5 \pm 2.5$ \\
Inter-day & & & \\
20 & $17.9 \pm 1.3$ & 7.6 & $89.3 \pm 6.7$ \\
40 & $38.6 \pm 3.5$ & 9.0 & $96.6 \pm 8.7$ \\
400 & $389.6 \pm 21.7$ & 5.6 & $97.4 \pm 5.4$ \\
750 & $748.2 \pm 7.8$ & 1.0 & $99.8 \pm 1.0$ \\
\hline
\end{tabular}

Data was shown as mean \pm standard deviation $(n=6)$.

\subsubsection{Stability and Dilution Integrity}

Methanolic stock solutions of FTS were found to remain stable for 3 months at $-70{ }^{\circ} \mathrm{C}$, with the mean stability ranging from $95.8 \%$ to $106.6 \%$. As shown in Table 2, the accuracy for FTS and ISTD in the plasma samples was within $15 \%$, which is acceptable and can be considered stable for $24 \mathrm{~h}$ within the autosampler [23]. Table 3 listed the stability results of benchtop and freeze-thaw tests. We have also evaluated the dilution integrity, and the results in Table 4, which indicated that the dilution steps have ignorable effects on the precision and accuracy.

Table 2. Stability of QC samples of FTS and ISTD in autosampler.

\begin{tabular}{cccc}
\hline \multirow{2}{*}{ Concentration (ng/mL) } & \multicolumn{3}{c}{ Time (h) } \\
\cline { 2 - 4 } & $\mathbf{0}$ & $\mathbf{1 6}$ & $\mathbf{2 4}$ \\
\hline 40 & 100 & $103.8 \pm 1.1$ & $102.4 \pm 9.0$ \\
400 & 100 & $104.8 \pm 1.8$ & $105.4 \pm 7.3$ \\
750 & 100 & $106.4 \pm 1.3$ & $107.1 \pm 2.7$ \\
2000 (ISTD) & 100 & $95.0 \pm 1.9$ & $96.3 \pm 4.4$ \\
\hline
\end{tabular}

Data was shown as percentage of peak area compared to the initial area as mean \pm standard deviation $(n=3)$. 
Table 3. Stability of FTS after freeze/thaw and benchtop storage conditions.

\begin{tabular}{ccccccc}
\hline \multirow{2}{*}{ Stability } & \multicolumn{2}{c}{ LQC (40 ng/mL) } & \multicolumn{2}{c}{ MQC (400 ng/mL) } & \multicolumn{2}{c}{ HQC (750 ng/mL) } \\
\cline { 2 - 6 } & Precision (\%) & Accuracy (\%) & Precision (\%) & Accuracy (\%) & Precision (\%) & Accuracy (\%) \\
\hline Benchtop for 8 h & 2.7 & $96.8 \pm 2.7$ & 6.3 & $99.4 \pm 6.3$ & 2.9 & $100.0 \pm 2.9$ \\
$\begin{array}{c}\text { Freeze/thaw } \\
\text { for 3 cycles }\end{array}$ & 6.7 & $96.1 \pm 6.4$ & 2.4 & $92.0 \pm 2.2$ & 5.3 & $106.1 \pm 5.6$ \\
\hline \multicolumn{7}{c}{ Data expressed as mean \pm standard deviation $(n=3)}$. \\
\hline
\end{tabular}

Table 4. Dilution integrity test for FTS analysis.

\begin{tabular}{ccccc}
\hline $\begin{array}{c}\text { Concentration in Spiked Rat } \\
\text { Plasma (ng/mL) }\end{array}$ & Diluted Times & Nominal Concentration (ng/mL) & Precision (\%) & Accuracy (\%) \\
\hline 1500 & 2 & 750 & 3.2 & $97.7 \pm 3.1$ \\
40,000 & 50 & 800 & 4.2 & $95.0 \pm 4.0$ \\
\hline
\end{tabular}

\subsection{Characterization of Hydrogel Formulations}

The temperature-viscosity and gel dissolution profiles of the seven formulations are shown in Figure S1. The $\mathrm{T}_{\text {sol-gel, }}$, gelling time, maximum viscosity, and complete dissolution time of the formulations are presented in Table 5. Formulation 1 (F1), simply containing F127, exhibited a $\mathrm{T}_{\text {sol-gel }}$ of approximately $26^{\circ} \mathrm{C}$. The additions of F68 and PEG 6000 both clearly increased $\mathrm{T}_{\text {sol-gel }}$ from $28{ }^{\circ} \mathrm{C}$ to $38^{\circ} \mathrm{C}$. F2 fortified with $1 \%(w / w)$ F68 had a gelling time of $1.4 \mathrm{~min}$, and F5 containing $0.5 \%(w / w)$ PEG 6000 had a gelling time of $1.7 \mathrm{~min}$. The increasing percentages of PEG 6000 in formulations decreased the maximum viscosities as reflected in F5 to F7, whereas F68 had limited effects on the maximum viscosities. F2 and F3 achieved complete dissolution around $96 \mathrm{~h}$ whereas other formulations needed $72 \mathrm{~h}$ or fewer.

A scoring criterion (Table S1) for the performance of hydrogel was developed to objectively judge the blank hydrogel formulations. By considering the importance of each physicochemical property such as $\mathrm{T}_{\text {sol-gel }}$, gelling time, maximum viscosity, and complete dissolution time, F2, F5, and F3 were selected for further FTS release evaluation. The accumulated FTS release profiles reached around $60 \%$ as shown in Figure 3A, and no difference was found among the three formulations (Figure 3A). The correlation coefficients between FTS release and gel dissolution were higher than 0.95 as shown in Figure 3B, indicating that the release was mostly controlled by the dissolution of the hydrogel [24]. The release kinetics results of FTS-loaded hydrogels (Table S2) suggested that the Higuchi model $\left(r^{2}=0.9114 \sim 0.9223\right)$ was more appropriate than were the zero-order model $\left(r^{2}=0.7153-0.7351\right)$ and the first-order kinetic model $\left(r^{2}=0.4066-0.4422\right)$.
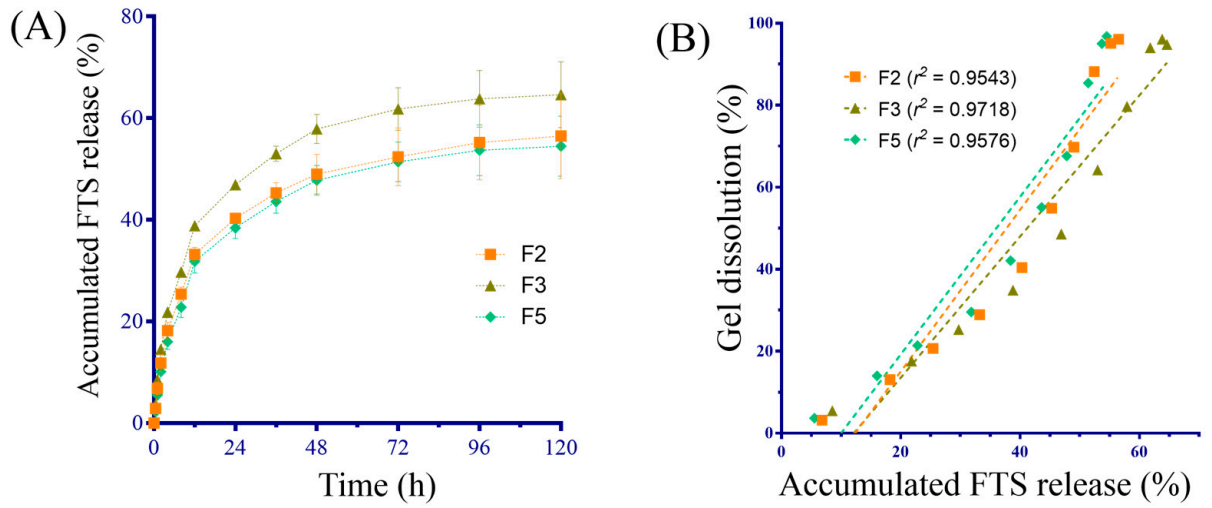

Figure 3. (A) The accumulative drug release rate of F2, F3 and F5 $(n=3)$; (B) Correlation profile of the accumulative percent of FTS released and the accumulative percent of thermo-sensitive hydrogel dissolved ( $n=3$ for each formulation). 
Table 5. Formulations, $\mathrm{T}_{\text {sol-gel }}$ and gelation time of FTS thermo-sensitive hydrogel.

\begin{tabular}{|c|c|c|c|c|c|c|c|c|c|}
\hline \multirow{2}{*}{ Formulation } & \multicolumn{3}{|c|}{ Composition } & \multirow{2}{*}{$\begin{array}{l}\text { Viscosity at } \\
26^{\circ} \mathrm{C}(\mathrm{cp})\end{array}$} & \multirow{2}{*}{$\mathrm{T}_{\text {sol-gel }}\left({ }^{\circ} \mathrm{C}\right)$} & \multirow{2}{*}{$\begin{array}{l}\text { Average Gelling } \\
\text { Time (min) }\end{array}$} & \multirow{2}{*}{$\begin{array}{c}\text { Maximum } \\
\text { Viscosity (cp) }\end{array}$} & \multirow{2}{*}{$\begin{array}{c}\text { Complete } \\
\text { Dissolution Time (h) }\end{array}$} & \multirow{2}{*}{ Total Score } \\
\hline & F127 (\%) & F68 (\%) & PEG $6000(\%)$ & & & & & & \\
\hline F1 & 17 & - & - & $1,266,000$ & 26 & 1.1 & $2 \times 10^{6}$ & 120 & N/A \\
\hline F2 & 17 & 1 & - & 138 & 28 & 1.4 & $2 \times 10^{6}$ & 96 & 15 \\
\hline F3 & 17 & 2 & - & 114 & 34 & 3.0 & $2 \times 10^{6}$ & 96 & 13 \\
\hline F4 & 17 & 3 & - & 102 & 36 & 4.5 & $2 \times 10^{6}$ & 72 & 6 \\
\hline F5 & 17 & - & 0.5 & 400 & 28 & 1.7 & $1.86 \times 10^{6}$ & 72 & 14 \\
\hline F6 & 17 & - & 1 & 120 & 30 & 1.6 & $1.33 \times 10^{6}$ & 72 & 12 \\
\hline F7 & 17 & - & 1.5 & 120 & 38 & 5.2 & $7.2 \times 10^{5}$ & 48 & 1 \\
\hline
\end{tabular}

N/A: Not applicable due to high viscosity at $26^{\circ} \mathrm{C}$. 


\subsection{Pharmacokinetic Evaluation of FTS}

The results of pharmacokinetic studies are listed in Figure 4 and Table 6 . The $t_{1 / 2}$ of subcutaneous (SC) FTS-loaded hydrogel (516.6 min) was significantly longer compared with the $t_{1 / 2}$ for free FTS (76.6 $\mathrm{min})$. In addition, the clearance $(\mathrm{Cl})$ of FTS-loaded hydrogel was $16.5 \mathrm{~mL} \cdot \mathrm{kg} / \mathrm{min}$, which is significantly lower than that of free FTS $(38.5 \mathrm{~mL} \cdot \mathrm{kg} / \mathrm{min})$. Finally, the hydrogel delivery system exhibited higher FTS bioavailability, as indicated by the AUC (161396 vs. $74894 \mathrm{~min} \cdot \mathrm{ng} / \mathrm{mL})$.

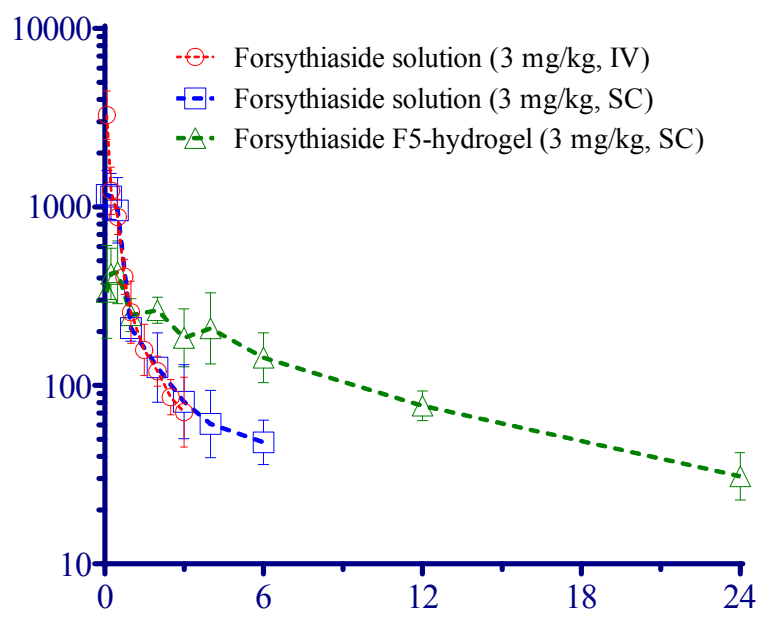

Figure 4. Concentration of forsythiaside in rat plasma vs. time curve after administration of drug (3 $\mathrm{mg} / \mathrm{kg}, n=4$ for each group).

Table 6. Pharmacokinetic parameters of FTS.

\begin{tabular}{ccccc}
\hline \multirow{2}{*}{ Parameter } & \multirow{2}{*}{ Unit } & \multicolumn{3}{c}{ Group } \\
\cline { 3 - 5 } & & 1 (Solution, IV) & 2 (Solution, SC) & 3 (F5-hydrogel, SC) \\
\hline $\mathrm{C}_{0}$ & $\mathrm{ng} / \mathrm{mL}$ & $36,385 \pm 10,387$ & $\mathrm{~N} / \mathrm{A}$ & $\mathrm{N} / \mathrm{A}$ \\
$\mathrm{C}_{\max }$ & $\mathrm{ng} / \mathrm{mL}$ & $\mathrm{N} / \mathrm{A}$ & $1518 \pm 162$ & $498 \pm 145^{*}$ \\
$\mathrm{t}_{1 / 2}$ & $\mathrm{~min}$ & $57.9 \pm 17.0$ & $76.6 \pm 7.2$ & $516.6 \pm 111.9^{*}$ \\
$\mathrm{AUC}_{0 \rightarrow \mathrm{t}}$ & $\mathrm{min} \mathrm{ng} / \mathrm{mL}$ & $149,462 \pm 32,852$ & $74,894 \pm 18,372$ & $161,396 \pm 21,771^{*}$ \\
$\mathrm{AUC} \mathrm{C}_{0 \rightarrow \infty}$ & $\mathrm{min} \mathrm{ng} / \mathrm{mL}$ & $155,726 \pm 29,172$ & $80,321 \pm 16,671$ & $184,856 \pm 28,599^{*}$ \\
$\mathrm{Cl}$ & $\mathrm{mL} \mathrm{kg/min}$ & $19.9 \pm 4.6$ & $38.5 \pm 7.6$ & $16.5 \pm 2.3^{*}$ \\
$\mathrm{~V}_{\mathrm{d}}$ & $\mathrm{mL} / \mathrm{kg}$ & $1683.6 \pm 669.6$ & $4261.0 \pm 953.8$ & $12,292.1 \pm 3333.1^{*}$ \\
$\mathrm{MRT}$ & $\mathrm{min}$ & $20.0 \pm 6.0$ & $75.5 \pm 7.8$ & $417.1 \pm 57.0^{*}$ \\
\hline
\end{tabular}

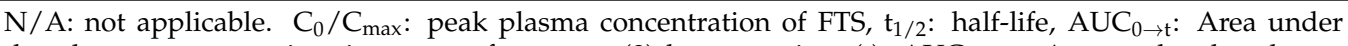
the plasma concentration-time curve from zero $(0)$ hours to time $(t), \mathrm{AUC}_{0 \rightarrow \infty}$ : Area under the plasma concentration-time curve from zero $(0)$ hours to infinity $(\infty)$, Cl: clearance, $V_{\mathrm{d}}$ : volume of distribution, MRT: mean residence time. Data was expressed with mean \pm standard deviation $(n=4) .{ }^{*} p<0.05$ compared with group 2 (Solution, SC).

\section{Discussion}

Botanic polyphenols possess diverse health-promoting benefits and their pharmacokinetic information has recently drawn much attention. Pharmacokinetic data can provide guidance for the safe and rational use of botanic and food supplements [25]. Analytical methods with high sensitivity facilitate the collection of more complete time-concentration profiles and improve the validity of the pharmacokinetic data. Table 7 listed liquid chromatographic methods of FTS for analytical performance comparison. EC is an appropriate choice for determining FTS concentrations, because EC provides higher selectivity and sensitivity than does UV; moreover, EC is not as susceptible to matrix effects as is MS. The potentials applied to the working electrode of EC were optimized to obtain the appropriate sensitivity for FTS. Higher potentials generally led to increased sensitivity as well as baseline noise 
and much longer equilibrium times. We therefore selected $+900 \mathrm{mV}$ to ensure adequate sensitivity while maintaining stable performance.

Table 7. Comparison of HPLC-based analytical method performance for forsythiaside.

\begin{tabular}{|c|c|c|c|c|c|}
\hline Detection Method & Sample Type & $\begin{array}{l}\text { Limit of Quantification } \\
\text { (ng/mL) }\end{array}$ & Linear Range (ng/mL) & $\underset{(\min )}{\operatorname{Run} \text { Time }}$ & Reference \\
\hline UV & Canine plasma & 52 & $52-13,330$ & 18 & [9] \\
\hline UV & Canine plasma & 78 & $78-40,000$ & 30 & [10] \\
\hline UV & Rat plasma & 67 & $67-26,667$ & 20 & [13] \\
\hline MS/MS & Rat plasma & 2.0 & $2.0-50.0$ and $50.0-5000.0$ & 10 & [2] \\
\hline MS/MS & Rat plasma & 2 & $2-500$ & 29 & [14] \\
\hline MS/MS & Rat plasma & 5.15 & $5.15-5150$ & 11 & [15] \\
\hline UPLC-MS/MS & Rat plasma & 0.46 & $0.46-236.10$ & 8 & [16] \\
\hline UPLC-MS/MS & Rat plasma & 0.4795 & $0.4795-982.1$ & 5.5 & [17] \\
\hline MS & Rat plasma & 1.294 & $1.294-2587.5$ & 10 & [26] \\
\hline $\mathrm{EC}$ & Rat plasma & 20 & 20-1000 & 30 & This study \\
\hline
\end{tabular}

FTS is a phenylethanoid glycoside with polar properties $(\log p=-0.5)$, which addresses the challenge of analysis in aqueous biological matrices [27]. To obtain adequate separation from the endogenous interference peaks, the composition of the mobile phase and the sample preparation methods were evaluated. Adjusting the $\mathrm{pH}(2.5,3.0,3.5$, and 4.0) of the mobile phase improved the FTS peak shape as a $\mathrm{pH}>3.5$ resulted in peak deterioration. Removing protein through denaturation and precipitation is a highly effective method for biological sample pretreatment and is commonly used for plasma and blood samples before analysis. Several precipitation reagents, including TCA $(10 \% w / v)$, perchloric acid (PCA, 6\% w/v), acetonitrile, acetone, and methanol, were evaluated. Significant interference peaks around the FTS elution time were found after precipitation by acetonitrile, acetone, and methanol. The addition of PCA resulted in the instability of FTS after $4 \mathrm{~h}$ at room temperature. Deproteinization using TCA was selected, which led to a region with minimal interference from 10 to $14 \mathrm{~min}$ (Figure 2A) and provided an acceptable recovery for FTS (approximately 80\%). Regarding detection and separation, we used a mobile phase composed of acetonitrile and phosphate solution (adjusted to $\mathrm{pH} 2.5$ with phosphoric acid) to give a retention time of $13.1 \mathrm{~min}$ for FTS. The developed HPLC-EC method has a 10-fold improvement in sensitivity compare with previously used HPLC-UV methods (LOQ $0.2 \mu \mathrm{g} / \mathrm{mL}$ ) [1,12].

The active compound releasing from hydrogel was affected by several factors such as the compound's partition between water and polymer, the water diffusion rate into the polymer, the drug diffusion rate from the hydrogel, and the hydrogel dissolution under our experimental conditions. To determine the drug release profile, the accumulative release ratio of FTS in hydrogel formulations (F2, F3 and F5) was fitted to various mathematical models, namely a zero-order kinetic model, a first-order kinetic model, and a Higuchi model. The coefficient of determination $\left(r^{2}\right)$ of the three formulations is listed in Table S2. The results of the mathematical models showed that the three formulations were best fitted by the Higuchi model $\left(r^{2}=0.9179,0.9114\right.$, and 0.9223 for F2, F3, and F5, respectively), indicating that the drug release followed Fickian diffusion [28]. The pharmacokinetic studies in rats demonstrated a significant $(p<0.05)$ difference in AUC $(2.1$ times) and mean residual time (5.5 times) between subcutaneous administration of FTS-loaded hydrogel and subcutaneous administration of FTS solutions. Studies have reported $t_{1 / 2}$ values of FTS after intravenously administration in rats around $73-77 \mathrm{~min}$ [2]. In this study, the $t_{1 / 2}$ was prolonged from 76.6 to $516.6 \mathrm{~min}$ after the subcutaneous administration of FTS-loaded hydrogel, which confirms the extended effect of the developed formulation. 


\section{Experimental Section}

\subsection{Chemicals and Reagents}

Forsythiaside (purity > 98\%) was obtained from Fusol Material Ltd. (Tainan, Taiwan). Pinoresinol (purity $>98 \%$ ) was acquired as internal standard (ISTD) from Seedchem (Melbourne, Australia). Puronic F127, F68, and PEG 6000 were purchased from Sigma-Aldrich (St. Louis, MO, USA). Methanol and acetonitrile (HPLC grade) were supplied by Tedia (Fairfield, OH, USA). Water was purified using the Milli-Q system (Millipore, Bedford, MA, USA). HPLC grade solvents were filtered through a 0.45- $\mu \mathrm{m}$ membrane filter (Millipore) and degassed in an ultrasonic bath (Branson Model 3210, Danbury, CT, USA) before use.

\subsection{Animals}

Male Sprague-Dawley (SD) rats (200 $\pm 20 \mathrm{~g}$ ) were purchased from BioLasco (Taipei, Taiwan). The animal experiment and caring protocol was reviewed and approved by the Institutional Animal Care and Use Committee of Kaohsiung Medical University (Approval number 102074). Animals were housed in standard laboratory conditions (temperature $25{ }^{\circ} \mathrm{C} \pm 2{ }^{\circ} \mathrm{C}$, relative humidity $50 \% \pm 20 \%$ ). Blood samples were collected at predetermined time points through jugular vein catheterization [29]. In brief, the rat was surgically implanted with a polyethylene tube (PE-50) in the right jugular vein. The PE-50 catheter was exteriorized, capped, and fixed in the dorsal neck region. The tubing patency was conserved by flushing with a normal saline solution $(0.9 \% \mathrm{NaCl}, w / v)$ containing heparin sodium $(15 \mathrm{IU} / \mathrm{mL})$.

\subsection{HPLC-EC Conditions}

Chromatographic analysis was conducted on a Chromaster HPLC system (Hitachi Ltd, Tokyo, Japan) consisting of a 5160 pump and a 5260 autosampler. FTS and IS were separated on a Kinetex XB-C18 column $(100 \mathrm{~mm} \times 2.1 \mathrm{~mm}$, i.d. $2.6 \mu \mathrm{m}$, Phenomenex, Torrance, CA, USA). The mobile phase comprised an acetonitrile-0.05-M phosphoric solution (11.8:88.2, v/v), and the $\mathrm{pH}$ was adjusted to 2.5 by using $O$-phosphoric acid. The mobile phase was delivered at $400 \mu \mathrm{L} / \mathrm{min}$ with an injection volume of $20 \mu \mathrm{L}$. The LC-4C electrochemical detector (Bioanalytical Systems, Inc. (BAS), West Lafayette, IN, USA) consists of a cross-flow cell equipped with a glassy carbon electrode (GCE, dia. $3 \mathrm{~mm} \times 2$ electrodes, dual type, ALS Co. Ltd., Tokyo, Japan), a Ag/AgCl reference electrode (RE-6 model, BAS), and a MF-1044 type gasket. A potential of $+900 \mathrm{mV}$ relative to the reference electrode was applied to the GCE by using a filter setting of $0.1 \mathrm{~Hz}$ and a range of $20 \mathrm{nA}$. Chromatographic results were acquired and processed using the Chromaster System Manager software (Tokyo, Japan).

\subsection{Preparation of Stock Solutions, Calibration Curves, and Quality Control Samples}

FTS and ISTD were individually dissolved in methanol to yield stock solutions at $100 \mu \mathrm{g} / \mathrm{mL}$ for each compound. The stock solutions were stored at $-70{ }^{\circ} \mathrm{C}$. The FTS stock solution was serially diluted with $50 \%(v / v)$ methanol to produce working standard solutions. A seven-point calibration curve was constructed by spiking $20 \mu \mathrm{L}$ of the working standard solution into $80 \mu \mathrm{L}$ blank plasma to obtain final concentrations of 20, 25, 50, 100, 200, 500, and $1000 \mathrm{ng} / \mathrm{mL}$. Samples of LQC, MQC and HQC were prepared in the same manner.

\subsection{Plasma Sample Pretreatment}

Ten percent $(w / v)$ trichloroacetic acid (TCA) was added to the plasma sample for deproteinization. The ISTD $(20 \mu \mathrm{L})$ was fortified into the plasma sample $(100 \mu \mathrm{L})$ in a $1.5-\mathrm{mL}$ plastic centrifuge vial, following which the TCA solution $(40 \mu \mathrm{L})$ was added and vortexed for $1 \mathrm{~min}$. The supernatant was kept after centrifugation $\left(14,000 \times g, 10 \mathrm{~min}, 4^{\circ} \mathrm{C}\right)$ and analyzed through HPLC-EC. 


\subsection{Method Validation}

\subsubsection{Selectivity}

Blank plasma samples from six different rats were prepared according to the aforementioned process. The selectivity was checked by inspecting interference peaks appearing around the retention times of FTS and ISTD.

\subsubsection{Linearity and Sensitivity}

The calibration curve was derived through least-square linear regression of the peak-area ratio versus FTS concentrations. Linearity was defined a coefficient of determination $\left(r^{2}\right)$ exceeding 0.995 . The LOQ was defined as the lowest concentration of the calibration curve with acceptable accuracy and precision. The LOD was defined as the concentration at a signal-to-noise ratio of 3:1 [29].

\subsubsection{Recovery}

The recovery of FTS after protein precipitation was calculated by comparing the peak areas of spiked samples to working standard samples with corresponding concentrations at 40,400, and $750 \mathrm{ng} / \mathrm{mL}(n=3)$. The recovery of ISTD was evaluated similarly.

\subsubsection{Accuracy and Precision}

LQC, MQC, and HQC samples were analyzed within 1 and 6 days, respectively, to evaluate intra- and interday accuracy and precision of the developed HPLC-EC method. Accuracy (\%) was expressed by dividing the measured concentration by the nominal concentration, and precision (\%) was expressed as the relative standard deviation (RSD).

\subsubsection{Stability}

Sample stability in the autosampler during HPLC analysis was evaluated at ambient temperature and at three concentrations (LQC, MQC and HQC) of samples analyzed after 0,16 , and $24 \mathrm{~h}$. The storage stability was evaluated by determining whether the stock solutions remained stable at $-70{ }^{\circ} \mathrm{C}$. Stock solutions at $0,1,2$, and 3 months were diluted with $50 \%(v / v)$ methanol to $400 \mathrm{ng} / \mathrm{mL}$ and analyzed through HPLC. The stability of benchtop and freeze/thaw for 3 cycles were also evaluated at three different concentrations (LQC, MQC and HQC).

\subsection{Preparation and of Physicochemical Evaluation of Hydrogel Formulations}

The procedures were established according to the cold method [30]. FTS was dispersed in different formulations containing F127 alone or with the addition of F68 or PEG 6000 at $4{ }^{\circ} \mathrm{C} \pm 2{ }^{\circ} \mathrm{C}$ under magnetic stirring. Each formulation was equilibrated in a $4{ }^{\circ} \mathrm{C}$ refrigerator until turning into a clear solution. The formulations are listed in Table 5. The $\mathrm{T}_{\text {sol-gel, }}$ viscosity, and gelling time of each formulation were measured using a DV2TLV viscometer (Brookfield, MA, USA) with a SC4-31 spindle, thermostatically controlled using a $\mathrm{B} 401 \mathrm{H}$ circulating water bath. Formulations were examined in the $10{ }^{\circ} \mathrm{C}-46^{\circ} \mathrm{C}$ range in increments of $2{ }^{\circ} \mathrm{C}$. After the set temperature was reached, a 5-min quiet time was observed before the viscosity test. When the viscosity increased 1000-fold, the temperature was recorded and defined as $\mathrm{T}_{\text {sol-gel }}$. The gelling time was defined as the time required to reach $50,000 \mathrm{cps}$ at $\mathrm{T}_{\text {sol-gel. }}$. The water bath was set at $37^{\circ} \mathrm{C} \pm 0.5^{\circ} \mathrm{C}$, and cold hydrogel $\left(4{ }^{\circ} \mathrm{C}\right)$ was tested.

The membrane-less method was used for hydrogel dissolution [24,31]. Each formulation $(4.0 \mathrm{~mL})$ was transferred into a conical glass test tube and was incubated for $30 \mathrm{~min}$ in a shaking water bath $\left(37^{\circ} \mathrm{C}, 70 \mathrm{rpm}\right)$ to solidify the hydrogel. An aliquot of $5 \mathrm{~mL}$ in phosphate buffered saline $(1.0 \mathrm{M}$, $\mathrm{pH} 7.4,37^{\circ} \mathrm{C}$ ) was added gently without disturbing the surface of the solidified gel. The dissolution medium was removed at predetermined time points, and the weight of the residual gel and test tube was measured. The residual gel in the test tube was refilled with fresh dissolution medium 
$(5.0 \mathrm{~mL})$ to continue dissolution. The loss of weight was calculated as the difference between the empty conical glass test tube and the tube with hydrogel to estimate the dissolution rate. The procedures for evaluating FTS release from F2, F3 and F5 were performed according to a previous report [24]. Dissolution samples were analyzed according to a previous method with appropriate modifications [13]. The data obtained from in vitro drug release studies of F2, F3, and F5 were further fitted to various mathematical models, including zero-order kinetic, first-order kinetic, and Higuchi model, to evaluate the drug release kinetics [28].

\subsection{Pharmacokinetic Evaluation}

Twelve SD rats were randomly divided into three groups ( $n=4$ in each group). The first group received $3 \mathrm{mg} / \mathrm{kg}$, IV FTS dissolved in $0.9 \% \mathrm{NaCl}$ through femoral vein catheterization. The second group received the same dose of FTS solution $(3 \mathrm{mg} / \mathrm{kg}$ ) through subcutaneous (SC) injection in the dorsal surface of the cervical region. The third group received the optimal hydrogel formulation $(3 \mathrm{mg} / \mathrm{kg})$ through subcutaneous (SC) injection. Blood samples were taken at predetermined time points, and plasma fractions were collected and stored at $-70{ }^{\circ} \mathrm{C}$ until analysis.

\subsection{Data Analysis}

Data were presented as means \pm standard deviation (STD). A standard noncompartmental pharmacokinetic model was used to calculate the following parameters $[32,33]$. The area under the curve (AUC) of plasma concentration versus time from zero to the last time point was calculated using the trapezoidal method. The mean retention time (MRT) was estimated as AUMC/AUC, where AUMC is the area under the first-moment curve. Clearance was calculated as dose/AUC, and the volume of distribution $\left(\mathrm{V}_{\mathrm{ss}}\right)$ was $\mathrm{Cl} \times$ MRT. Finally, half-life $\left(\mathrm{t}_{1 / 2}\right)$ was determined at $\ln 2 / \mathrm{K}_{\mathrm{e}}$. Comparison of the data was assessed through one-way analysis of variance and followed by Fisher's least significant difference by using the SPSS software (IBM SPSS Statistics 14.0). $p<0.05$ was considered significant.

\section{Conclusions}

A sensitive and validated HPLC-EC method was developed for quantifying FTS in rat plasma. The validation results indicated that this method has good linearity from 20 to $1000 \mathrm{ng} / \mathrm{mL}$, with $r^{2}>0.999$. An efficient deproteination method using TCA achieved FTS recovery exceeding 79\%. Precision and accuracy varied by less than $10 \%$, indicating the reliability and repeatability of the method. The method was applied to the in vivo pharmacokinetic evaluation of FTS-TIGS. The pharmacokinetic results showed a significant extension of the FTS residual time using our hydrogel formulation as evidenced by the significantly increased AUC and $t_{1 / 2}$, as well as the reduced $\mathrm{Cl}$ of FTS.

Supplementary Materials: The following are available online at http://www.mdpi.com/1420-3049/21/10/ $1384 / \mathrm{s} 1$.

Acknowledgments: Funding for this study was provided in part by research grants KMU-TP104A47 from Kaohsiung Medical University and MOST103-2113-M-037-004-MY2 from the Ministry of Science and Technology, Taiwan.

Author Contributions: Y.-T.W. conceived and designed the experiments; Y.-T.W., M.-T.C. and C.-C.Y. performed the experiments; Y.-T.W., C.-W.C., and M.-C.H. analyzed the data; Y.-T.W. and M.-T.C. wrote the paper.

Conflicts of Interest: The authors declare no conflict of interest.

\section{References}

1. Chu, Y.; Wang, X.; Guo, J.; Li, W.; Ma, X.; Zhu, Y. Pharmacokinetic study of unbound forsythiaside in rat blood and bile by microdialysis coupled with HPLC method. Eur. J. Drug Metab. Pharmacokinet. 2012, 37, 173-177. [CrossRef] [PubMed]

2. Wang, G.-N.; Pan, R.-L.; Liao, Y.-H.; Chen, Y.; Tang, J.-T.; Chang, Q. An LC-MS/MS method for determination of forsythiaside in rat plasma and application to a pharmacokinetic study. J. Chromatogr. B 2010, 878, 102-106. [CrossRef] [PubMed] 
3. Sun, H.; Liu, M.; Lin, Z.; Jiang, H.; Niu, Y.; Wang, H.; Chen, S. Comprehensive identification of 125 multifarious constituents in Shuang-Huang-Lian powder injection by HPLC-DAD-ESI-IT-TOF-MS. J. Pharm. Biomed. Anal. 2015, 115, 86-106. [CrossRef] [PubMed]

4. Cheng, X.-R.; Zhou, W.-X.; Zhang, Y.-X. The behavioral, pathological and therapeutic features of the senescence-accelerated mouse prone 8 strain as an Alzheimer's disease animal model. Ageing Res. Rev. 2014, 13, 13-37. [CrossRef] [PubMed]

5. Ko, H.-C.; Wei, B.-L.; Chiou, W.-F. The effect of medicinal plants used in Chinese folk medicine on RANTES secretion by virus-infected human epithelial cells. J. Ethnopharmacol. 2006, 107, 205-210. [CrossRef] [PubMed]

6. Qu, H.; Zhang, Y.; Chai, X.; Sun, W. Isoforsythiaside, an antioxidant and antibacterial phenylethanoid glycoside isolated from Forsythia suspense. Bioorg. Chem. 2012, 40, 87-91. [CrossRef] [PubMed]

7. Schenone, M.; Dancik, V.; Wagner, B.K.; Clemons, P.A. Target identification and mechanism of action in chemical biology and drug discovery. Nat. Chem. Biol. 2013, 9, 232-240. [CrossRef] [PubMed]

8. Hirtz, J. Importance of analytical methods in pharmacokinetic and drug metabolism studies. Biopharm. Drug Dispos. 1986, 7, 315-326. [CrossRef] [PubMed]

9. Shi, R.; Xuan, Z.; Ma, Y.; Liu, Y.; Lu, H.; Sun, T. Pharmacokinetics of forsythoside after intravenous administration in beagle dogs. Eur. J. Drug Metab. Pharmacokinet. 2009, 34, 101-105. [CrossRef] [PubMed]

10. Li, Y.X.; Peng, C.; Zhang, R.Q.; Li, X.; Jiang, X.H. Pharmacokinetics of phillyrin and forsythiaside following IV administration to Beagle dog. Eur. J. Drug Metab. Pharmacokinet. 2009, 34, 79-83. [CrossRef] [PubMed]

11. Li, Y.-X.; Peng, C.; Zhang, R.-Q.; Li, X.; Jiang, X.-H. Simultaneous Determination of Phillyrin and Forsythiaside in Beagle Dog Plasma by High-Performance Liquid Chromatography with Gradient Elution and Its Application to Pharmacokinetic Studies. Anal. Lett. 2010, 43, 209-218. [CrossRef]

12. Tian, J.; Lin, L.; Li, X.; Dou, H.; Pei, W.; Wang, L.; Han, J.; Ni, J.; Zheng, H. Forsythiaside stability in pretreated rat plasma and its pharmacokinetics after iV. administration. Anal. Methods 2015, 7, 1809-1815. [CrossRef]

13. Li, Y.-X.; Jiang, X.-H.; Liang, H.-Y.; Li, X. Determination of forsythiaside in rat plasma by high-performance liquid chromatography and its application to pharmacokinetic studies. Biomed. Chrom. 2008, 22, 361-366. [CrossRef] [PubMed]

14. Bai, Y.; Li, J.; Liu, W.; Jiao, X.-C.; He, J.; Liu, J.; Ma, L.; Gao, X.-M.; Chang, Y.-X. Pharmacokinetic of 5 components after oral administration of Fructus forsythiae by HPLC-MS/MS and the effects of harvest time and administration times. J. Chromatogr. B 2015, 993-994, 36-46. [CrossRef] [PubMed]

15. Ye, J.-X.; Wei, W.; Quan, L.-H.; Liu, C.-Y.; Chang, Q.; Liao, Y.-H. An LC-MS/MS method for the simultaneous determination of chlorogenic acid, forsythiaside $\mathrm{A}$ and baicalin in rat plasma and its application to pharmacokinetic study of Shuang-Huang-Lian in rats. J. Pharm. Biomed. Anal. 2010, 52, 625-630. [CrossRef] [PubMed]

16. Zhou, W.; Tam, K.Y.; Meng, M.; Shan, J.; Wang, S.; Ju, W.; Cai, B.; Di, L. Pharmacokinetics screening for multi-components absorbed in the rat plasma after oral administration of traditional Chinese medicine Flos Lonicerae japonicae-Fructus forsythiae herb couple by sequential negative and positive ionization ultra-high-performance liquid chromatography/tandem triple quadrupole mass spectrometric detection. J. Chromatogr. A 2015, 1376, 84-97. [PubMed]

17. Zhou, W.; Shan, J.; Wang, S.; Ju, W.; Meng, M.; Cai, B.; Di, L. Simultaneous determination of caffeic acid derivatives by UPLC-MS/MS in rat plasma and its application in pharmacokinetic study after oral administration of Flos lonicerae-Fructus forsythiae herb combination. J. Chromatogr. B 2014, 949-950, 7-15. [CrossRef] [PubMed]

18. Ismaiel, O.A.; Halquist, M.S.; Elmamly, M.Y.; Shalaby, A.; Karnes, H.T. Monitoring phospholipids for assessment of matrix effects in a liquid chromatography-tandem mass spectrometry method for hydrocodone and pseudoephedrine in human plasma. J. Chromatogr. B Anal. Technol. Biomed. Life Sci. 2007, 859, 84-93. [CrossRef] [PubMed]

19. Wu, Y.-T.; Tsai, T.-R.; Lin, L.-C.; Tsai, T.-H. Liquid chromatographic method with amperometric detection to determine acteoside in rat blood and brain microdialysates and its application to pharmacokinetic study. J. Chromatogr. B 2007, 853, 281-286. [CrossRef] [PubMed]

20. Kempe, S.; Mäder, K. In situ forming implants-An attractive formulation principle for parenteral depot formulations. J. Control. Release 2012, 161, 668-679. [CrossRef] [PubMed]

21. Gupta, P.; Vermani, K.; Garg, S. Hydrogels: From controlled release to pH-responsive drug delivery. Drug Discov. Today 2002, 7, 569-579. [CrossRef] 
22. Ahmed, E.M. Hydrogel: Preparation, characterization, and applications: A review. J. Adv. Res. 2015, 6, 105-121. [CrossRef] [PubMed]

23. Kumar, M.; Sharma, G.; Singla, D.; Singh, S.; Sahwney, S.; Chauhan, A.S.; Singh, G.; Kaur, I.P. Development of a validated UPLC method for simultaneous estimation of both free and entrapped (in solid lipid nanoparticles) all-trans retinoic acid and cholecalciferol (vitamin D3) and its pharmacokinetic applicability in rats. J. Pharm. Biomed. Anal. 2014, 91, 73-80. [CrossRef] [PubMed]

24. Radivojša, M.; Grabnar, I.; Ahlin Grabnar, P. Thermoreversible in situ gelling poloxamer-based systems with chitosan nanocomplexes for prolonged subcutaneous delivery of heparin: Design and in vitro evaluation. Eur. J. Pharm. Sci. 2013, 50, 93-101.

25. Van Breemen, R.B.; Fong, H.H.; Farnsworth, N.R. Ensuring the safety of botanical dietary supplements. Am. J. Clin. Nutr. 2008, 87, 509S-513S. [PubMed]

26. Zhou, W.; Di, L.-Q.; Shan, J.-J.; Bi, X.-L.; Chen, L.-T.; Wang, L.-C. Intestinal absorption of forsythoside A in different compositions of Shuang-Huang-Lian. Fitoterapia 2011, 82, 375-382. [CrossRef] [PubMed]

27. Kovalova, L.; McArdell, C.S.; Hollender, J. Challenge of high polarity and low concentrations in analysis of cytostatics and metabolites in wastewater by hydrophilic interaction chromatography/tandem mass spectrometry. J. Chromatogr. A 2009, 1216, 1100-1108. [CrossRef] [PubMed]

28. Nasir, F.; Iqbal, Z.; Khan, J.A.; Khan, A.; Khuda, F.; Ahmad, L.; Khan, A.; Khan, A.; Roohullah, A.D. Development and evaluation of diclofenac sodium thermorevesible subcutaneous drug delivery system. Int. J. Pharm. 2012, 439, 120-126. [CrossRef] [PubMed]

29. Chang, C.-W.; Wong, C.-Y.; Wu, Y.-T.; Hsu, M.-C. Development of a Solid Dispersion System for Improving the Oral Bioavailability of Resveratrol in Rats. Eur. J. Drug Metab. Pharmacokinet. 2016. [CrossRef] [PubMed]

30. Schmolka, I.R. Artificial skin I. Preparation and properties of pluronic F-127 gels for treatment of burns. J. Biomed. Mater. Res. 1972, 6, 571-582. [PubMed]

31. Xie, B.; Jin, L.; Luo, Z.; Yu, J.; Shi, S.; Zhang, Z.; Shen, M.; Chen, H.; Li, X.; Song, Z. An injectable thermosensitive polymeric hydrogel for sustained release of Avastin ${ }^{\circledR}$ to treat posterior segment disease. Int. J. Pharm. 2015, 490, 375-383. [CrossRef] [PubMed]

32. Gibaldi, M.; Perrier, D. Multicompartment models. In Pharmacokinetics; Marcel Dekker, Inc: New York, NY, USA, 1982; pp. 45-112.

33. Ragelle, H.; Crauste-Manciet, S.; Seguin, J.; Brossard, D.; Scherman, D.; Arnaud, P.; Chabot, G.G. Nanoemulsion formulation of fisetin improves bioavailability and antitumour activity in mice. Int. J. Pharm. 2012, 427, 452-459. [CrossRef] [PubMed]

Sample Availability: Samples of the working standard solution of forsythiaside are available from the authors.

(C) 2016 by the authors; licensee MDPI, Basel, Switzerland. This article is an open access article distributed under the terms and conditions of the Creative Commons Attribution (CC-BY) license (http://creativecommons.org/licenses/by/4.0/). 